Linköping Studies in Science and Technology

Dissertations No. 1830

\title{
Small-amplitude steady water waves with vorticity
}

Evgeniy Lokharu

Department of Mathematics, Division of Mathematics and Applied Mathematics

Linköping University, SE-581 83 Linköping, Sweden

Linköping 2017 
Linköping Studies in Science and Technology. Dissertations No. 1830 Small-amplitude steady water waves with vorticity

Copyright (C) Evgeniy Lokharu, 2017

Division of Mathematics and Applied Mathematics

Department of Mathematics

Linköping University

SE-581 83, Linköping, Sweden

evgeniy.lokharu@liu.se

ISSN 0345-7524

ISBN 978-91-7685-587-4

Printed by LiU-Tryck, Linköping, Sweden 2017 
To my wife Svetlana 



\begin{abstract}
The problem of describing two-dimensional traveling water waves is considered. The water region is of finite depth and the interface between the region and the air is given by the graph of a function. We assume the flow to be incompressible and neglect the effects of surface tension. However we assume the flow to be rotational so that the vorticity distribution is a given function depending on the values of the stream function of the flow. The presence of vorticity increases the complexity of the problem and also leads to a wider class of solutions.

First we study unidirectional waves with vorticity and verify the Benjamin-Lighthill conjecture for flows whose Bernoulli constant is close to the critical one. For this purpose it is shown that every wave, whose slope is bounded by a fixed constant, is either a Stokes or a solitary wave. It is proved that the whole set of these waves is uniquely parametrised (up to translation) by the flow force which varies between its values for the supercritical and subcritical shear flows of constant depth. We also study largeamplitude unidirectional waves for which we prove bounds for the free-surface profile and for Bernoulli's constant.

Second, we consider small-amplitude waves with counter currents. Such flows admit layers, where the fluid flows in different directions. In this case we prove that the initial nonlinear free-boundary problem can be reduced to a finite-dimensional Hamiltonian system with a stable equilibrium point corresponding to a uniform stream. As an application of this result, we prove the existence of non-symmetric wave profiles. Furthermore, using a different method, we prove the existence of periodic waves with an arbitrary number of crests per period.
\end{abstract}




\section{Populärvetenskaplig sammanfattning}

I denna avhandling betraktar vi problemet att beskriva tvådimensionella vandrande vattenvågor. Vattenregionen antas ha ändligt djup och ytan mellan området och luften beskrivs av grafen till en funktion. Vi antar att flödet är inkompressibelt and bortser från ytspänningseffekter. Vidare antar vi att flödet är inte är rotationsfritt, så att vorticiteten beskrivs av en given funktion som beror på värden av strömmningsfunktionen för flödet. Existensen av vorticitet ökar komplexiteten hos problemet och leder också till en större lösningsklass.

Först studerar vi enkelriktade vågor med vorticitet och verifierar Benjamin-Lighthillkonjekturen för flöden vars Bernoullikonstant ligger nära den kritiska nivån. Av denna anledning visas att varje våg vars lutning är begränsad av en fix konstant är antingen en Stokesvåg eller en solitär våg. Hela mängden av dessa vågor kan på ett entydigt sätt parametriseras (upp till translationer) av flödeskraften som varierar mellan värden för superkritiska och subkritiska skjuvflöden på konstant djup. Vi studerar också enkelriktade vågor med hög amplitud där vi visar begränsningar för den fria ytprofilen och för Bernoullikonstanten.

Sedan betraktar vi lågamplitudsvågor med motströmmar. Sådana flöden tillåter lager där vätskan flödar i olika riktningar. För detta fall visar vi att det ickelinjära fria initialrandvärdesproblemet kan reduceras till ett ändligtdimensionellt Hamiltonskt system med en stabil jämviktspunkt motsvarande en likformig strömmning. Som en tillämpning av detta resultat bevisar vi existensen av icke-symmetriska vågprofiler. Dessutom, genom en annan metod, bevisar vi existensen av periodiska vågor med ett godtyckligt antal toppar per period. 


\section{Acknowledgements}

First I would like to express my sincere gratitude to my supervisor Vladimir Kozlov. I am indebted to him for his excellent guidance and advice, for his patience and continuous support. I thank Vladimir for his encouragement and for being a great teacher for me, for his invaluable contribution to my mathematical education.

I am grateful to my co-supervisors Johan Thim and Tomas Sjödin for their continuous support, advice and encouragement. I would like to express my sincere appreciation to my co-author Nikolai Kuznetsov for his invaluable help and interesting discussions. My deepest thanks to Vladimir Tkatjev for his kind support and encouragements.

I thank all my colleagues at the Department of Mathematics for creating a pleasant working atmosphere. Especially I am grateful to my friends Arpan, Sonja, Anna, Samira, David, Alexandra, Jolanta, Zhenxia, Xiangfeng, Armen and Spartak.

Finally, I thank my family who always supported and believed in me.

Evgeniy Lokharu Linköping, January 19, 2017 


\section{List of Papers}

The following papers are included in this thesis and will be referred to by their roman numerals.

I V. Kozlov, N. Kuznetsov, E. Loharu, On bounds and non-existence in the problem of steady waves with vorticity, Journal of Fluid Mechanics 765, 2015.

II V. Kozlov, N. Kuznetsov, E. Loharu, On the Benjamin\&Lighthill conjecture for water waves with vorticity, submitted to Journal of Fluid Mechanics.

III V. Kozlov, E. Loharu, $N$-modal steady water waves with vorticity, manuscript.

IV V. Kozlov, E. Loharu, Small-amplitude steady water waves with critical layers: nonsymmetric waves, manuscript.

In all papers I contributed by doing all the derivations. In papers (i) and (ii) I wrote all the mathematical part, while Vladimir Kozlov and Nikolay Kuznetsov contributed by discussions and exposition. In papers (iii) and (iv) all the work on writing the papers was done by me, while Vladimir Kozlov contributed by discussions and writing two subsections.

The papers that will not be included in thesis are as follows:

1. V. Kozlov, N. Kuznetsov, E. Loharu, Steady water waves with vorticity: An analysis of the dispersion equation, Journal of Fluid Mechanics 751, 2014. 


\section{Contents}

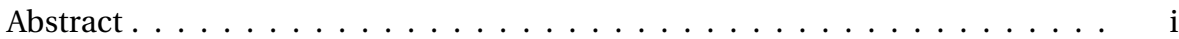

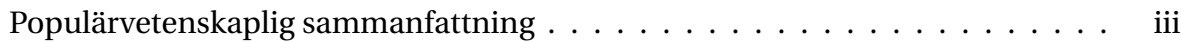

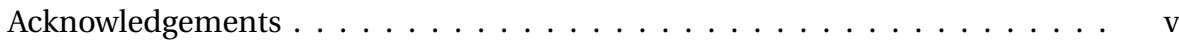

List of Papers . . . . . . . . . . . . . . . . . . vii

Introduction $\quad 1$

1 Mathematical model for the gravity-driven water waves of finite depth 3

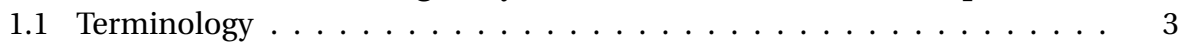

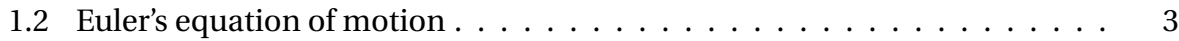

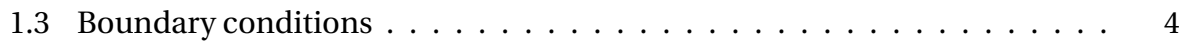

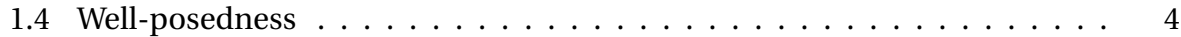

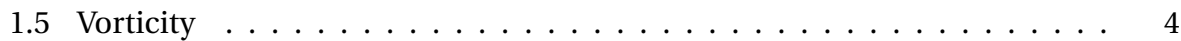

2 Two-dimensional steady waves with vorticity $\mathbf{5}$

2.1 Stream function formulation $\ldots \ldots \ldots \ldots \ldots$

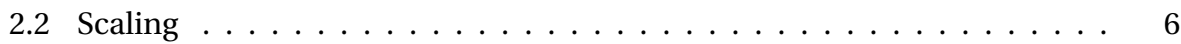

2.3 The structure of the discussion of the thesis $\ldots \ldots \ldots \ldots$

3 Unidirectional waves with vorticity 7

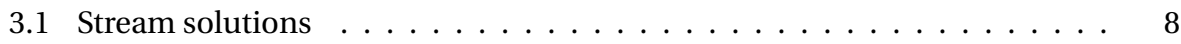

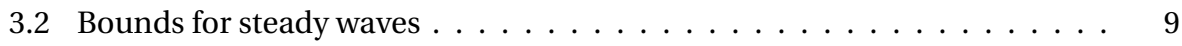

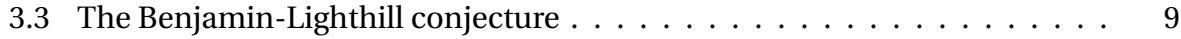

4 Steady waves over streams with counter-currents $\quad 10$

4.1 Linear approximation of the problem and the dispersion equation . . . . . 11

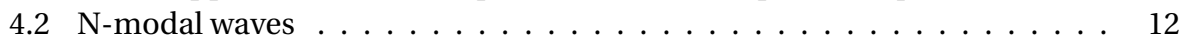

4.3 Non-symmetric waves . . . . . . . . . . . . . . . . . 13

$\begin{array}{ll}\text { Bibliography } & 14\end{array}$

Paper I: On bounds and non-existence in the problem of steady waves $\begin{array}{ll}\text { with vorticity } & 18\end{array}$ Paper II: On the Benjamin\&Lighthill conjecture for water waves with
vorticity

Paper III: N-modal steady water waves with vorticity 
Paper IV: Small-amplitude steady water waves with critical layers: non-symmetric waves 


\section{Introduction}





\section{Mathematical model for the gravity-driven water waves of finite depth}

In the three-dimensional space with the coordinates $X, Y, Z$, we consider a region of a liquid, say water, bounded from above by the graph of a function $\hat{\eta}(X, Y, Z)$. The bottom of the region is flat and coincides with the level $Y=0$, while the gravity acts in the negative $Y$-direction. Let $\mathbf{u}$ be the velocity field of the flow. In order to simplify mathematical model, we make several assumptions regarding the fluid:

- Constant density. In particular, this implies that the fluid is incompressible. In terms of the velocity field it reads as

$$
\mathbf{u}_{X}+\mathbf{u}_{Y}+\mathbf{u}_{Z}=0
$$

everywhere in the fluid. For simplicity we will assume that the density $\rho=1$.

- No surface tension. It is reasonable to neglect the effects of surface tension for waves that are not very small.

- No viscosity.

- Air pressure is constant so that we neglect air motion above the fluid region.

\subsection{Terminology}

By a surface wave or just wave we mean the top boundary of the fluid domain. By assumption it coincides with the graph of a function - surface profile. We will often identify a wave with the corresponding profile function. By a flow we understand a motion of the fluid. If the surface of the fluid region is flat, then the corresponding flow is called a current. Since we are only concerned with the two-dimensional steady flows, we will assume in what follows that the velocity field of a current depends only on the $Y$-coordinate. We will often use terms "irrotational waves", "unidirectional waves" or "rotational waves" when talking about waves over flows with a certain property specified by the corresponding adjective.

\subsection{Euler's equation of motion}

Let $\mathbf{X}(t)$ be the position of a fluid particle at time $t$. Then the velocity field along the path is given by $\mathbf{u}(\mathbf{X}(t), t)$. Applying Newton's law of motion to a fluid particle, we find

$$
\frac{\partial[\mathbf{u}(\mathbf{X}(t), t)]}{\partial t}=-\frac{1}{\rho} \nabla P+(0,-g, 0),
$$

where $\rho$ is the density constant and $P$ stands for the hydrodynamic pressure. Thus, everywhere in the fluid, we have

$$
\frac{D \mathbf{u}}{D t}=-\frac{1}{\rho} \nabla P+(0,-g, 0),
$$


where the operator $D / D t$ is called total derivative and is defined via

$$
\frac{D}{D t}=\frac{\partial}{\partial t}+\mathbf{u} \cdot \nabla
$$

The latter equation (1.2) is called Euler's equation of motion.

\subsection{Boundary conditions}

Euler's equation (1.2) is naturally equipped by two types of boundary conditions: kinematic and dynamic. The kinematic boundary conditions express the fact that fluid particles can not leave the fluid domain:

$$
u_{2}=\eta_{t}+u_{1} \eta_{X}+u_{3} \eta_{Z} \text { for } Y=\hat{\eta}(X, Z)
$$

and

$$
u_{2}=0 \text { for } Y=0 .
$$

Since in the present model we neglect the surface tension and the air pressure is assumed to be constant, we obtain the following dynamic boundary condition:

$$
P=P_{a t m} \text { for } Y=\hat{\eta}(X, Z) \text {. }
$$

Euler's equation (1.2) together with (1.1) and the boundary conditions (1.3), (1.4), (1.5) are called the governing equations for water waves. The unknowns are the surface profile $\hat{\eta}$, pressure $P$ and velocity field $\mathbf{u}$.

\subsection{Well-posedness}

We say that the problem is locally well-posed if for a proper initial data for $t=0$ the following is true: (i) a solution to the problem exists in some interval of time $[0, T]$; (ii) the solution is unique; (iii) the solution depends continuously on the initial data. It was proved in [26] that the problem for irrotational waves is locally well-posed, where an irrotational flow is characterized by the identity $\operatorname{curl}(\mathbf{u})=0$. In the general case wellposedness is established (see [9], [27]) only under additional assumptions on the initial data.

\subsection{Vorticity}

An important characteristic of a flow is the vorticity, which measures the local rotation of a fluid particle. In terms of the velocity field it is defined by

$$
\boldsymbol{\omega}=\operatorname{curl}(\mathbf{u})=\nabla \times \mathbf{u} .
$$


Taking the curl in Euler's equation, one can show that the vorticity $\omega$ satisfies

$$
\frac{D \omega}{D t}=(\boldsymbol{\omega} \cdot \nabla) \mathbf{u}
$$

The latter equation implies that if a fluid particle has zero vorticity at some point, then it is zero everywhere along the particle's path.

If $\boldsymbol{\omega}=0$ everywhere in the fluid, we say that the flow (or wave) is irrotational. Otherwise, we have a rotational flow or a rotational wave.

\section{Two-dimensional steady waves with vorticity}

In the present thesis we study only two-dimensional travelling waves defined as follows. Let us assume that all the functions $\mathbf{u}, P, \hat{\eta}$ are independent of the $Z$-coordinate. Furthermore, we assume that there is a coordinate system moving with a constant speed $c$ in negative $X$-direction in which all the functions are independent of time. In particular, this means that $\hat{\eta}(X, Z, t)=\eta_{0}(X-c t)$ for some function $\eta_{0}$. Thus, when time goes, the profile of a wave just shifts in the $x$-direction without changing its shape. Similarly, we have

$$
\mathbf{u}(X, t)=\mathbf{u}_{0}(X, X-c t), \quad \omega(X, Y, t)=\omega_{0}(X, Y, X-c t), \quad P(X, Y, t)=P_{0}(X, Y, X-c t) .
$$

Let us define the domain

$$
D_{0}=\left\{(X, Y) \in \mathbb{R}^{2}: 0<Y<\eta_{0}(X)\right\}
$$

In the new moving frame, equations (1.2) and (1.1) transforms into

$$
\begin{aligned}
& (u-c) u_{X}+v u_{Y}=-\left(P_{0}\right)_{X}, \\
& (u-c) v_{X}+v v_{Y}=-\left(P_{0}\right)_{Y}-g, \quad \text { in } D_{0} \\
& u_{X}+u_{Y}=0 .
\end{aligned}
$$

Here $\mathbf{u}_{0}=(u, 0, v)$. The boundary conditions (1.3), (1.4), (1.5) become

$$
\begin{aligned}
& v=(u-c)\left(\eta_{0}\right)_{X}, \quad Y=\eta_{0}(X), \\
& v=0, \quad Y=0, \\
& P_{0}=P_{a t m}, \quad Y=\eta_{0}(X) .
\end{aligned}
$$

Furthermore, a direct calculation shows that $\omega_{0}=\left(0, \omega_{0}, 0\right)$, where

$$
\omega_{0}(X, Y)=v_{X}-u_{Y}
$$




\subsection{Stream function formulation}

Given two points $\left(X_{0}, Y_{0}\right),(X, Y) \in D_{0}$, we put

$$
\Psi(X, Y)=\int_{\left(X_{0}, Y_{0}\right)}^{(X, Y)}(u-c) d Y-v d X .
$$

Since $u_{X}+v_{Y}=0$ the latter definition is independent of the path of integration. The function $\Psi$ is called a stream function and satisfies

$$
\Psi_{X}=-v, \Psi_{Y}=u-c .
$$

Let us write the governing equations in terms of the stream function. Because $\omega_{0}(X, Y)=$ const along every level set $\Psi(X, Y)=p$, we assume that $\omega_{0}$ depends only on the values $\Psi(X, Y)$. Thus, we obtain

$$
\begin{aligned}
& \Psi_{X X}+\Psi_{Y Y}+\omega_{0}(\Psi)=0, \text { in } D_{0} \\
& \Psi=m_{1} \text { on } Y=\eta_{0}(X), \\
& \Psi=m_{0} \text { on } Y=0, \\
& \Psi_{X}^{2}+\Psi_{Y}^{2}+2 g Y=Q \text { on } Y=\eta_{0}(X) .
\end{aligned}
$$

where $m_{1}$ and $m_{0}$ are some constants such that

$$
m_{1}-m_{0}=\int_{0}^{\eta_{0}(X)}(u-c) d Y .
$$

Since the function $\Psi$ is defined up to a constant, we can assume that $m_{0}=0$. The last equation (2.4) represents the dynamic boundary condition. The constant $Q$ is called the total head.

\subsection{Scaling}

From mathematical point of view it is convenient to use the non-dimensional variables proposed by Keady and Norbury [17]. Namely, lengths and velocities are scaled to $\left(m_{1}^{2} / g\right)^{1 / 3}$ and $\left(m_{1} g\right)^{1 / 3}$ respectively. More precisely, assuming $m_{1} \neq 0$, we introduce new coordinates

$$
x=\lambda X, \quad y=\lambda Y
$$

and new functions defined by

$$
\psi(x, y)=m_{1} \Psi(X, Y), \eta(x)=\lambda \eta_{0}(x / \lambda), \omega(p)=\left(m_{1} \lambda^{2}\right)^{-1} \omega_{0}\left(m_{1} p\right),
$$

where

$$
\lambda=\left(\frac{g}{m_{1}^{2}}\right)^{1 / 3} .
$$


Hence, the problem (2.1)-(2.4) is equivalent to

$$
\begin{aligned}
& \psi_{x x}+\psi_{y y}+\omega(\psi)=0, \text { in } D \\
& \psi=1 \text { on } y=\eta(x), \\
& \psi=0 \text { on } y=0, \\
& \psi_{x}^{2}+\psi_{y}^{2}+2 y=3 r \text { on } y=\eta(x),
\end{aligned}
$$

where $D=\left\{(x, y) \in \mathbb{R}^{2}: 0<y<\eta(x)\right\}$ and the constant $r$, referred to as Bernoulli's constant, is defined by

$$
r=\frac{Q}{3\left(m_{1} \lambda\right)^{2}}
$$

\subsection{The structure of the discussion of the thesis}

In order to discuss the results of the thesis it is convenient to distinguish the following three directions in the theory of steady waves:

- irrotational waves;

- unidirectional waves;

- waves over flows with counter-currents or waves with critical layers;

Papers I and II contain results on unidirectional waves, while in Papers III and IV we study waves with counter-currents. The next section is dedicated to unidirectional waves and provides all necessary definitions and a short overview of the field. In Subsection 3.2, we discuss Paper I and Section 3.3 is concerned with Paper II. In Section 4 we discuss waves which are not necessarily unidirectional and explain the contribution of Papers III and IV.

\section{Unidirectional waves with vorticity}

We say that a flow with stream function $\psi$ is unidirectional, if

$$
\psi_{y}>0 \text { in } D .
$$

The first existence result on unidirectional waves is due to Dubreil-Jacotin [8], who constructed small-amplitude Stokes waves using power series expansions. The main invention of [8] was the use of the partial hodograph transform:

$$
D \ni(x, y) \mapsto(q, p) \in S=\mathbb{R} \times(0,1) .
$$

We consider $(q, p)$ as independent variables in $S$ and we use $y$ as the new unknown function for which $h(q, p)$ is the standard notation. A straightforward calculation shows that

$$
h_{q}=-\frac{\psi_{x}}{\psi_{y}} \quad \text { and } \quad h_{p}=\frac{1}{\psi_{y}}
$$


thus yielding that problem (2.5)-(2.8) takes the following form in terms of the new variables:

$$
\begin{aligned}
& \left(\frac{h_{q}}{h_{p}}\right)_{q}-\frac{1}{2}\left(\frac{1+h_{q}^{2}}{h_{p}^{2}}\right)_{p}-\omega(p)=0, \quad(q, p) \in S \\
& h(0, q)=0, \quad q \in \mathbb{R} ; \\
& \frac{1+h_{q}^{2}}{h_{p}^{2}}=3 r-2 h, \quad p=1, q \in \mathbb{R} .
\end{aligned}
$$

The advantage of this formulation is twofold. First we obtain the problem with only one unknown function of two variables. Secondly the domain is fixed, while the operator on the left-hand side in (3.2) is elliptic.

In 2004 Constantin and Strauss published the famous paper [6] that initiated an intensive research on steady flows with vorticity. Using a bifurcation argument and degree theory, they constructed small- and large-amplitude Stokes waves with vorticity. The existence of unidirectional solitary waves of small amplitude was established in [14] and [16] independently using different methods. In [14] the authors use a spatial dynamics approach to characterize all small-amplitude waves for near-critical values of the Bernoulli constant (these are only Stokes and solitary waves). On the other hand, the argument in [16] is based on an implicit function theorem of Nash-Moser type. Largeamplitude solitary waves were constructed in [34].

\subsection{Stream solutions}

By a stream solution (shear flow), we mean a pair $(u(y), d)$, where $u$ stands for the stream function instead of $\psi$ and the constant depth of flow $d$ replaces the wave profile $\eta$. It follows from (3.2)-(3.3) that a unidirectional stream solution in terms of the heightfunction $H(q, p)$ is subject to

$$
\left(\frac{1}{H_{p}^{2}}\right)_{p}=2 \omega(p), \quad p \in(0,1) ; H(0)=0 .
$$

Thus, for any $\lambda>\lambda_{*}$ there is a unique solution $H(p ; \lambda)$ such that $H_{p}(0)=1 / \lambda$, provided

$$
\lambda_{*}=\sqrt{2 \max _{0 \leq \tau \leq 1} \Omega(\tau)}, \quad \text { where } \Omega(\tau)=\int_{0}^{\tau} \omega(t) d t .
$$

The corresponding Bernoulli's constant and the depth are given by

$$
r=\mathscr{R}(\lambda), \text { where } \mathscr{R}(\lambda)=\left[\lambda^{2}-2 \Omega(1)+2 d(\lambda)\right] / 3
$$

and

$$
d(\lambda)=\int_{0}^{1} \frac{d \tau}{\sqrt{\lambda^{2}-2 \Omega(\tau)}} .
$$


The function $\mathscr{R}(\lambda)$ is convex and attains its minimum for some $\lambda=\lambda_{c}>\lambda_{*}$. The value $r_{c}=\mathscr{R}\left(\lambda_{c}\right)$ will be referred to as the critical value of the Bernoulli constant. Furthermore, for a given $r \in\left(r_{c}, r_{*}\right)$ with $r_{*}=\mathscr{R}\left(\lambda_{*}\right)$ there are exactly two roots $\lambda_{+}<r_{c}<\lambda_{-}$of the equation $\mathscr{R}(\lambda)=r$. These roots correspond to the stream solutions $\left(u_{+}, d_{+}\right)$and $\left(u_{-}, d_{-}\right)$, called subcritical and supercritical flows respectively. The notation is chosen so that $d_{-}<d_{+}$.

It should be noted that flows with zero vorticity are necessarily unidirectional so that the definitions above make sense. Thus, if $\Omega(p)=0$ for all $p \in[0,1]$, we have $\lambda_{*}=0$ and $r_{*}=\infty$.

\subsection{Bounds for steady waves}

In Paper I we obtain bounds for the surface profile and Bernoulli's constant of an arbitrary unidirectional steady wave. First, we show that the inequality

$$
r>r_{c}
$$

is valid for an arbitrary unidirectional steady wave which is sufficiently smooth. The estimate (3.6) for irrotational solitary waves was obtained independently by Amick \& Toland [2] and McLeod [28], while Kozlov and Kuznetsov [18] treated the general case of zero vorticity. Recently, Wheeler [35] proved it for unidirectional solitary waves.

In the second part of Paper I we prove bounds for the profile. Let $(\psi, \eta)$ be a solution of the problem (2.5)-(2.8) with $r \in\left(r_{c}, r_{0}\right)$ and such that $\psi_{y} \geq \delta$ in $D$ for some $\delta>0$. Then we have

$$
\sup _{x \in \mathbf{R}} \eta(x) \geq d_{+}(r)>\inf _{x \in \mathbf{R}} \eta(x) \text { and } \eta(x)>d_{-}(r)
$$

for all $x \in \mathbb{R}$. Finally, in the case $r>r_{0}$, we show that under some assumptions on the vorticity there are no solutions of the problem other than uniform streams. Similar results was obtained in [20] for flows with counter-currents under certain restrictions.

\subsection{The Benjamin-Lighthill conjecture}

Before formulating the conjecture, we will give some definitions. Following [17], we introduce the flow force invariant as follows:

$$
s(\psi, \eta)=\left[r+\frac{2}{3} \Omega(1)\right] \eta(x)-\frac{1}{3}\left\{\eta^{2}(x)-\int_{0}^{\eta(x)}\left[\psi_{y}^{2}-\psi_{x}^{2}-2 \Omega(\psi)\right] d y\right\} .
$$

Here $\Omega(p)=\int_{0}^{p} \omega(t) d t$. It is easy to check that the expression above is independent of $x$ and so is an invariant of the flow. Calculating the flow force invariant for the suband supercritical flows we obtain values $s_{+}(r)$ and $s_{-}(r)$ respectively. Let us define the cuspidal region

$$
\mathscr{C}=\left\{(r, s): r>r_{c}, s_{-}(r) \leq s \leq s_{+}(r)\right\} .
$$

The boundary of $\mathscr{C}$ consists of two parts $\Gamma_{ \pm}=\left\{\left(r, s_{ \pm}(r)\right), r \geq r_{c}\right\}$ corresponding to subcritical and supercritical uniform streams respectively. 
In 1954 Benjamin \& Ligthill [4] conjectured the following regarding irrotational steady flows:

(a) Every steady wave is represented by a point in $\mathscr{C}$.

(b) To every point in $\mathscr{C}$ corresponds some steady wave.

(c) The correspondence between steady waves and points in $\mathscr{C}$ is one-to-one.

We shall make a comment about assertion (c). First, we note that for any solitary wave with Bernoulli's constant $r$ the flow force coincides with $s_{-}(r)$, which follows from (3.7) by passing to the limit. Thus, supercritical flows and solitary waves have the same representation in $\mathscr{C}$. Therefore, we shall consider the correspondence between all nonuniform flows and the region $\mathscr{C} \backslash \Gamma_{-}$.

Assertion (a) for irrotational Stokes waves was verified by Keady \& Norbury [17] (see also Benjamin [3]). Assertion (c) fails in general. In 1992 Plotnikov [32] proved that for some $r>r_{c}$ there exist two distinct solitary waves and since for any solitary wave with Bernoulli's constant $r$ the flow force coincides with $s_{-}(r)$, we find that assertion (c) is false. Furthermore, numerical results obtained by Cokelet [5] show that assertion (b) is not true, at least for Stokes waves. On the other hand, Kozlov \& Kuznetsov [21] proved that both assertions (a) and (b) for irrotational flows are true in a neighbourhood of the singular point $\left(r_{c}, s_{c}\right)$, that is if $r$ is close to its critical value $r_{c}$.

In Paper II we are concerned with the proof of the Benjamin-Lighthill conjecture for unidirectional waves. There are two central results in [25]. First, we prove Theorem 4 which states that any sufficiently smooth solution of the problem with bounded slopes is of small amplitude, provided the Bernoulli constant is close to its critical value. In the irrotational case a stronger result is valid (see [21,22]) without any assumptions on the slopes. The rest of Paper II deals with the verification of the conjecture: we prove assertions (a), (b) and (c) for near-critical $r$ in the class of solutions with bounded slopes.

\section{Steady waves over streams with counter-currents}

From the present understanding of unidirectional waves it is seen that these waves have similar properties as irrotational waves. So far only Stokes and solitary waves are known in both classes (relatively to the problem for steady waves of finite depth and without surface tension). However, once we omit assumption (3.1) for unidirectional waves, we obtain a much more complicated class of solutions. The first existence result in this direction was obtained by Wahlén [33] in 2009. The author considers the case of constant vorticity and proves the existence of small-amplitude periodic waves with critical layers which are regions of the fluid consisting of entirely closed streamlines. In [7] Constantin \& Varvaruca treat a similar problem but allowing overhanging profiles. In [11] and [12] the authors consider linear vorticity distributions and construct bimodal waves with several crests in each minimal period. The first approximation for such waves is given by a combination of two cos-functions with different periods. In [1] the authors, 
using different bifurcation parameters, construct bimodal waves that are different from those obtained in [11] and [12]. Later, in 2015, Ehrnström and Wahlén [13] established existence of trimodal waves. These are periodical waves of small amplitude with the first approximation given by a combination of three basic modes (cos-functions with different periods).

There are also other results on steady waves without assumption (3.1). A detailed classification of currents is given in [23], while local bifurcation theory of Stokes waves over flows with counter-currents is constructed in [24].

\subsection{Linear approximation of the problem and the dispersion equation}

Let us consider a formal linear approximation of the problem (2.5)-(2.8). For this purpose, we rectify the domain $D$ by the following transformation

$$
z=y \frac{d}{\eta(x)} \text {. }
$$

Thus, the domain $D$ transforms into the strip $S=\mathbb{R} \times(0, d)$ and instead of the stream function $\psi(x, y)$, we consider

$$
\Phi(x, z)=\psi\left(x, y \frac{\eta(x)}{d}\right) .
$$

Since we are interested in small perturbations of a stream solution, we put

$$
\Phi(x, z)=u(z)+\epsilon \hat{\Phi}(x, z)+o\left(\epsilon^{2}\right), \eta(x)=d+\epsilon \zeta(x)+o\left(\epsilon^{2}\right) .
$$

Using this ansatz in (2.5)-(2.8), we obtain the following equations for the couple $(\hat{\Phi}, \zeta)$ which give the first-order approximation of the solution:

$$
\begin{aligned}
& {\left[\hat{\Phi}_{x}-\frac{z \zeta_{x} u_{z}}{d}\right]_{x}+\hat{\Phi}_{z z}-\frac{2 \zeta u_{z z}}{d}+\omega^{\prime}(u) \hat{\Phi}=0,} \\
& \hat{\Phi}(x, 0)=\hat{\Phi}(x, d)=0, \\
& \left.\hat{\Phi}_{z}\right|_{z=d}-\left(\frac{u^{\prime}(d)}{d}-\frac{1}{u^{\prime}(d)}\right) \zeta=0 .
\end{aligned}
$$

The latter problem can be essentially simplified by letting

$$
\Psi=\hat{\Phi}-\frac{z \zeta u_{z}}{d} .
$$

This formula implies $\zeta=-\left.\Psi\right|_{z=d} / u^{\prime}(d)$ and then the linear part of the above problem transforms into

$$
\begin{aligned}
& \Psi_{x x}+\Psi_{z z}+\omega^{\prime}(u) \Psi=0 \\
& \left.\Psi\right|_{z=0}=0 \\
& \left.\Psi_{z}\right|_{z=d}-\left.\kappa \Psi\right|_{x=d}=0,
\end{aligned}
$$


where $\kappa=1 /\left[u^{\prime}(d)\right]^{2}-\omega(1) / u^{\prime}(d)$. Separation of variables in the system (4.1) leads to the following Sturm-Liouville problem:

$$
-\phi_{z z}-\omega^{\prime}(u) \phi=\mu \phi \text { on }(0, d), \quad \phi(0)=0, \quad \phi_{z}(d)=\kappa \phi(d) .
$$

This eigenvalue problem (4.2) will be referred to as the dispersion equation. The spectrum of (4.2) depends only on the triple $(\omega, d, u)$ and consists of a countable set of simple real eigenvalues $\left\{\mu_{j}\right\}_{j=1}^{\infty}$ ordered so that $\mu_{j}<\mu_{l}$ for all $j<l$. Furthermore, only a finite number of the eigenvalues may be negative.

Solving the linear problem (4.1), we find that the space of bounded solutions is finitedimensional and is spanned by the functions

$$
\cos \left(\sqrt{\left|\mu_{j}\right|} x\right) \phi_{j}(z), \sin \left(\sqrt{\left|\mu_{j}\right|} x\right) \phi_{j}(z)
$$

for all $\mu_{j} \leq 0$, where $\phi_{j}$ is the corresponding eigenfunction.

In both Papers III and IV, we study small-amplitude waves over flows with countercurrents. In contrast to unidirectional waves, the dispersion equation may have any number of roots (see [10]), which means that the linear problem (4.1) possesses bounded periodic solutions with different wavelengths. A natural question here is about justification of the linear approximation given by (4.1): are there nonlinear solutions to the problem (2.5) - (2.8) for which the linear approximations are given by those solving the linear problem (4.1). It turned out that this standard problem is far away from being easy. In Papers III and IV, we provide an approach to this problem.

\section{$4.2 \quad \mathrm{~N}$-modal waves}

In the papers [11], [1], [13] the authors construct symmetric small-amplitude and periodic waves for which the linear approximation is given by a combinations of two or three co-sinus functions with different wavelengths. In [11] they state a natural question: are there waves for which the linear approximation is given by any number of basic modes? In Paper III we affirmatively answer this question.

The main difficulty for the higher order bifurcation is the fact that the number of natural parameters of the problem is limited. Normally, one may consider only the Bernoulli constant or the wavelength as bifurcation parameters. In the case of linear vorticity, the derivative of the vorticity function (which is a constant in this case) is another possible parameter.

In our approach, we use roots of the dispersion equation as bifurcation parameters. For this purpose we study the following inverse spectral problem (see Theorem 3.1 in Paper III): for a given $\mu=\left(\mu_{1}, \ldots, \mu_{N}\right) \in \mathbf{R}^{N}$ find a vorticity function $\omega(p ; \mu)$ and a stream solution $(u(y ; \mu), d)$ such that all negative eigenvalues of (4.2) for the triple $(\omega, d, u)$ coincide with $\mu_{1}, \ldots, \mu_{N}$, while all others are positive. We solve this problem for $\mu \in \Gamma$, where $\Gamma$ is an open set in $\mathbb{R}^{N}$. The bifurcation value $\mu^{*}=\left(\mu_{1}^{*}, \ldots, \mu_{N}^{*}\right) \in \Gamma$ is chosen so that $\mu_{j}^{*} / \mu_{l}^{*}$ are rational numbers for all $1 \leq j, l \leq N$ and $u_{z}\left(d ; \mu_{*}\right) \neq 0$. In this case all solutions to the 
linear problem (4.1) for the triple $\left(\omega\left(p ; \mu_{*}\right), d, u\left(z ; \mu_{*}\right)\right)$ have a common period $\Lambda_{*}$. The main result of Paper III is:

Theorem 1 (Existence of $\mathrm{N}$-modal waves). Under the given assumptions there exist constants $\epsilon, \delta>0$ depending only on $N$ and $d$ and a smooth function $\mu:[-\delta, \delta]^{N} \rightarrow \Gamma$ with the following property: for any $t:=\left(t_{1}, \ldots, t_{N}\right) \in \mathbb{R}^{N}$ such that $|t|<\delta$ and $|t|^{2} / t_{j} \leq \epsilon$ the nonlinear water wave problem (2.5)-(2.8) with the vorticity $\omega(p ; \mu(t))$ possesses a unique $\Lambda_{*}$ periodic and even solution $(\psi, \eta)$ corresponding to the Bernoulli constant $r(t)=\left[u_{z}(d ; \mu(t))^{2}+\right.$ $2 d] / 3$ such that

$$
\eta(x)=d+t_{1} \cos \left(\sqrt{\left|\mu_{1}^{*}\right| x}\right)+\ldots+t_{N} \cos \left(\sqrt{\left|\mu_{N}^{*}\right|} x\right)+O\left(|t|^{2}\right) .
$$

The solutions constructed in the theorem are even in the $x$-variable and this is essential for our argument.

\subsection{Non-symmetric waves}

Similar to Paper III, we study small-amplitude waves, assuming that the dispersion equation has several roots. In contrast to Paper III, we do not assume solutions to be even and in this case the linear problem (4.1) may possess (see [10] for the proof) non-symmetric solutions as a combination of sinus and co-sinus functions with different wavelengths. Are there non-symmetric small-amplitude solutions to the initial nonlinear problem (2.5) - (2.8)? In Paper IV, we affirmatively answer this question and prove that in a certain sense the most of small-amplitude solutions are non-symmetric (see Theorem 2 below).

Our method is based on an implementation of a spatial dynamics approach ([14, 15, $29,31,36]$ ). First, we formulate the problem as an evolutionary equation for which the role of time is played by the spatial coordinate. Then, using a center manifold reduction technique due to Mielke [30], we reduce the problem to a finite-dimensional system of ordinary differential equations with a definite first integral given by the flow force invariant. This implies that the equilibrium point corresponding to a uniform stream is stable. As a result, we obtain a large class of small-amplitude solutions containing non-symmetric steady waves.

It is worth to mention that the reduced system obtained in Paper IV is Hamiltonian for which the Hamiltonian is given by the flow force invariant (see [19]).

Let us formulate the main result of Paper IV. For any $N>1$ let $\omega \in C^{m+1}(\mathbb{R})$ for some $m \geq 1$ and $(u, d)$ be a stream solution corresponding to $\omega$ and such that (i) $u^{\prime}(d) \neq 0$; (ii) the Sturm-Liouville problem (4.2) has exactly $N$ non-positive egenvalues $\mu_{1}<\ldots<\mu_{N} \leq$ 0. According to Theorem 3.1 in Paper IV, the problem (2.5) - (2.8) for small-amplitude waves is reduced to the following system:

$$
\begin{aligned}
& \alpha_{j}^{\prime}=\beta_{j}+f_{1 j}(\alpha, \beta) \\
& \beta_{j}^{\prime}=-\left|\mu_{j}\right| \alpha_{j}+f_{2 j}(\alpha, \beta), j=1, \ldots, N .
\end{aligned}
$$

Here $\alpha, \beta \in C^{m}(\mathbb{R})$ and the remainders $f_{1 j}, f_{2 j}$ are smooth and consist of quadratic and lower order terms. Now we can formulate 
Theorem 2. Under the given assumptions the following statements are true:

(i) there exists an open neighbourhood $W$ of the origin in $\mathbb{R}^{2 N}$ such that the reduced system (4.3) possesses a unique solution $(\alpha, \beta)$ for any initial data $(\alpha(0), \beta(0)) \in W$;

(ii) let $W^{\text {sym }}$ be the set of all $(p, q) \in W$ such that there exists a solution $(\alpha, \beta)$ which is symmetric around some vertical line $x=x_{0}$ and such that $(p, q)=(\alpha(t), \beta(t))$ for some $t \in \mathbb{R}$. Then the Hausdorff dimension of $W^{\text {sym }}$ is less than or equal to $N+1$.

Now it follows from the theorem that for any $N>1$ there are non-symmetric solutions.

\section{Bibliography}

[1] Aasen, A. , Varholm, K. Traveling water waves with critical layers. arXiv:1508.04664 (2015).

[2] Amick, C. J., Toland, J. F. On periodic water-waves and their convergence to solitary waves in the long-wave limit. Phil. Trans. R. Soc. Lond. A 303, 633-669 (1981).

[3] Benjamin, T. B. Verification of the Benjamin-Lighthill conjecture about steady water waves. J. Fluid Mech. 295, 337-356 (1995).

[4] Benjamin, T. B., Lighthill,M. J. On cnoidal waves and bores. Proc. R. Soc. Lond. A224, 448-460.

[5] Cokelet, E. D. Steep gravity waves in water of arbitrary uniform depth. Phil. Trans. R. Soc. Lond. A 286, 183-230 (1977).

[6] Constantin, A., Strauss W., Exact steady periodic water waves with vorticity. Comm. Pure Appl. Math. 57, 481-527 (2004).

[7] Constantin, A., Varvaruca, E.: Steady periodic water waves with constant vorticity: regularity and local bifurcation. Arch. Rat. Mech. Anal. 199, 33-67 (2011).

[8] Dubreil-Jacotin, M.L.: Sur la détermination rigoureuse des ondes permanentes périodiques d?ampleur finie. J. Math. Pures Appl. 9(13), 217-291 (1934).

[9] Coutand, D., Shkoller, S. Well-posedness of the free-surface incompressible Euler equations with or without surface tension, J. Amer. Math. Soc. 20, 829-930 (2007).

[10] Ehrnström, M., Holden, H., Raynaud, X. Symmetric waves are traveling waves. Int. Math. Res. Not., 4686-4708 (2009).

[11] Ehrnström, M., Escher, J., Wahlén, E. Steady water waves with multiple critical layers. SIAM J. Math. Anal. 43(3), 1436?1456 (2011)

[12] Ehrnström, M., Escher, J., Villari, G. Steady water waves with multiple critical layers: interior dynamics. J. Math. Fluid Mech. 14(3), 407--419 (2012) 
[13] Ehrnström, M., Wahlén, E. Trimodal Steady Water Waves, Arch. Ration. Mech. Anal. 216, no. 2, 449-471 (2015).

[14] Groves, M.D., Wahlén, E. Small-amplitude Stokes and solitary gravity water waves with an arbitrary distribution of vorticity, Physica D: Nonlinear Phenomena, Volume 237, Issues 10?12, 1530-1538 (2008).

[15] Groves M.D., Wahlén, E. Spatial Dynamics Methods for Solitary Gravity-Capillary Water Waves with an Arbitrary Distribution of Vorticity, SIAM J. Math. Anal., 39(3), 932-964.

[16] Hur, V.-M. Exact Solitary Water Waves with Vorticity, Archive for Rational Mechanics and Analysis, Volume 188, Issue 2, pp 213--244 (2008).

[17] Keady, G., Norbury, J. Waves and conjugate streams with vorticity. Mathematika 25, 129-150 (1978).

[18] Kozlov, V., Kuznetsov, N. Fundamental bounds for arbitrary steady water waves. Math. Ann. 345, 643-655 (2009).

[19] Kozlov, V., Kuznetsov, N. Steady water waves with vorticity: spatial Hamiltonian structure. Journal of Fluid Mechanics, Vol. 733 (2013).

[20] Kozlov, V., Kuznetsov, N. Bounds for steady water waves with vorticity. J. Differential Equations 252, 663-691 (2012).

[21] Kozlov, V., Kuznetsov, N. The Benjamin-Lighthill conjecture for near-critical values of Bernoulli's constant. Arch. Rat. Mech. Anal. 197, 433-488 (2010).

[22] Kozlov, V., Kuznetsov, N. The Benjamin-Lighthill Conjecture for Steady Water Waves (revisited). Archive for Rational Mechanics and Analysis, Volume 201, Issue 2, 631-645 (2011).

[23] Kozlov, V., Kuznetsov, N. Steady free-surface vortical flows parallel to the horizontal bottom. Quart. J. Mech. Appl. Math. 64, 371-399 (2011).

[24] Kozlov, V., Kuznetsov, N. Dispersion Equation for Water Waves with Vorticity and Stokes Waves on Flows with Counter-Currents. Archive for Rational Mechanics and Analysis, Volume 214, Number 3 (2014).

[25] Kozlov, V., Kuznetsov, N., Lokharu, E. On the Benjamin-Lighthill conjecture for water waves with vorticity, to appear.

[26] Lannes, D. Well-posedness of the water-waves equations, J. Amer. Math. Soc. 18, 605--654 (2005).

[27] Lindblad, H. Well-posedness for the motion of an incompressible liquid with free surface boundary, Ann. of Math. (2) 162, 109--194 (2005). 
[28] McLeod, J. B. The Froude number for solitary waves. Proc. R. Soc. Edinburgh A 97, 193-197 (1984).

[29] Mielke A. Reduction of quasilinear elliptic equations in cylindrical domains with applications. Math. Methods Appl. Sci., 10, 51--66, (1988).

[30] Mielke, A. Hamiltonian and Lagrangian Flows on Center Manifolds, SpringerVerlag, Berlin (1991).

[31] Mielke, A. A Reduction Principle for Nonautonomous Systems in InfiniteDimensional Spaces. Journal Of Differential Equations 65, 68-88, (1986).

[32] Plotnikov, P.I. Non-uniqueness of solution of the problem of solitary waves and bifurcation of critical points of smooth functionals. Math. USSR Izvestia 38, 333357 (1992).

[33] Wahlén, E. Steady water waves with a critical layer. J. Differ. Equ. 246(6), 2468—2483 (2009).

[34] Wheeler, M. H. Large-Amplitude Solitary Water Waves with Vorticity, SIAM Journal on Mathematical Analysis, Vol. 45, No. 5 : pp. 2937-2994 (2013).

[35] Wheeler, M. H. The Froude number for solitary water waves with vorticity. J. Fluid Mech. 768, 91-112 (2015).

[36] Buffoni, B.; Groves, M. D.; Toland, J. F. A plethora of solitary gravity-capillary water waves with nearly critical Bond and Froude numbers. Philos. Trans. Roy. Soc. London Ser. A 354, no. 1707, 575--607 (1996). 


\section{Papers}

The articles associated with this thesis have been removed for copyright reasons. For more details about these see:

http://urn.kb.se/resolve? urn:nbn:se:liu:diva-134243 